R. J. Cohen and W. T. Sullivan, III, eds.

\title{
RFI Sentinel 2
}

\author{
S. Montebugnoli, M. Cecchi, C. Bortolotti, M. Roma and S. Mariotti \\ Radio Astronomy Institute, National Research Council, Bologna, Italy
}

\begin{abstract}
Nowadays we have a massively increasing use of radio techniques in a wide variety of application fields. Meanwhile state-of-the-art receiver technology dramatically increases the sensitivity of modern radio telescopes. This situation produces a worrying vulnerability of groundbased radio telescopes to Radio Frequency Interference (RFI). In order to monitor the RFI scenario within the frequency bands reserved for radio astronomy activities, a monitoring system, based on a quite new approach, has been developed and is presented here.
\end{abstract}

\section{The System}

The main characteristic of the "Sentinel 2" system is to perform an FFT analysis on a down-converted band up to $20 \mathrm{MHz}$ wide, step-tuned under programmed control over a broader frequency range. This approach, unlike that of high-cost commercial sweeping receivers, allows the system to achieve fast scanning while maintaining high frequency resolution.

The system architecture, along with the designed post-processing tools, offers the possibility to deal with different kinds of interfering weak signals independently of whether they are stationary, moving or burst-like. A hypothetical commercial receiver (i.e $80-\mathrm{kHz}$ frequency resolution) might take 23,750 steps to sweep a 0.1-2.0 GHz band. Since each step takes time, the RFI scenario obtained could be very different from the real situation. Both rapidly variable interference and spread spectrum signals are, generally, missed (Figure 1). Scanning the same range with a wideband window $(20 \mathrm{MHz})$ it is possible to compute a power spectrum over a programmable number of channels (i.e. 256 ) maintaining a similar resolution but drastically decreasing the number of steps (in this case by a factor of 250). If the FFT computation is fast enough, a good RFI scenario, much closer to the real one, is obtained (Figure 2).

The signal coming from the sweeping receiver is digitized with a 12-bit analogue-to-digital (A/D) converter after a proper downconversion to the video band (theoretically 12-bits should allow a 72-dB dynamic range). Data are acquired in burst mode and transferred (via PCI bus) to the PC memory (500-MHz Pentium III powered), then they are block-processed for the spectral analysis. Since the power spectral computation takes time, the analysis is not in real time. In fact, while it is in progress, the A/D converter is stopped, introducing a "hole" in the acquisition beside every working cycle. The resulting duty cycle is, anyway, acceptable because the system is not requested to work in real time. 


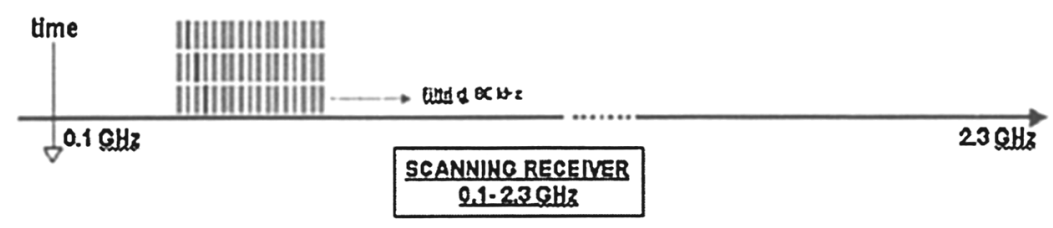

Figure 1. Commercial receiver's working mode.

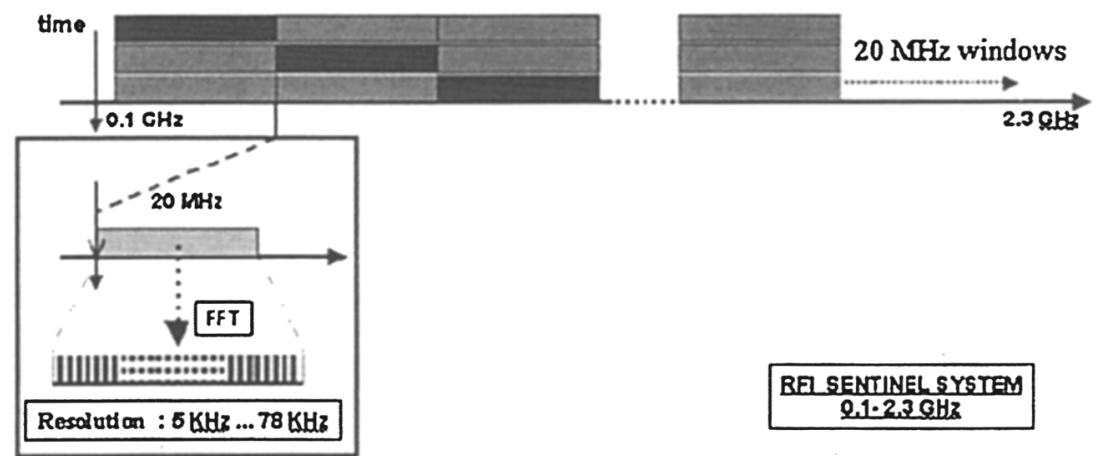

Figure 2. Sentinel 2 working mode.

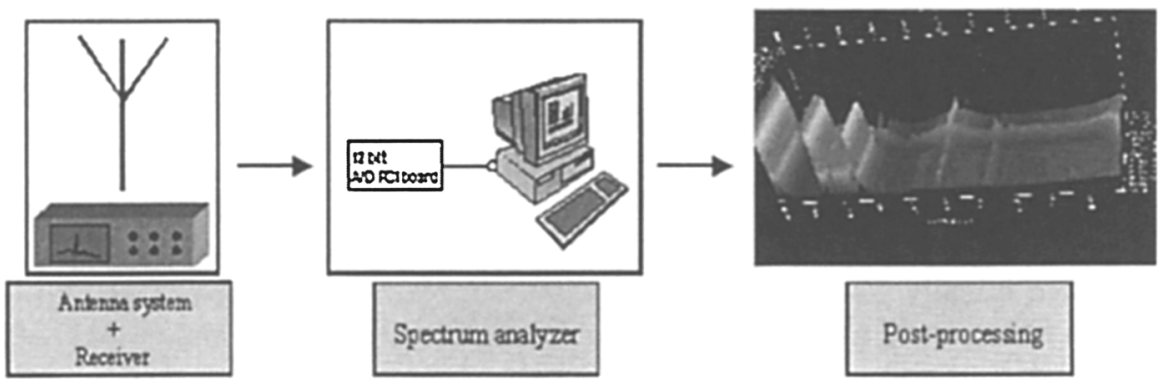

Figure 3. Sentinel 2 System Block Diagram. 
The system architecture is shown in Figure 3. Each working cycle is composed, in more detail, of the following steps:

- data acquisition in a burst;

- data transfer from the ADC board buffer to the PC memory;

- complex spectrum computation (Radix 2) for every burst series;

- power spectrum and average computation;

- data storage on a SCSI 2 hard disc;

- local oscillator and antenna selection control (IEEE 488 bus);

- data-base management and display.

The time taken to perform the above steps in the case of bursts of 256, 128, 64 spectra is given in the following table ${ }^{1}$ :

\begin{tabular}{|l|l|l|r|r|r|}
\hline \hline Channels & Resolution & Mode & 256 & 128 & 64 \\
\hline 256 & $78 \mathrm{kHz}$ & 1 work-cycle (ms) & 2,57 & 3,91 & 6,34 \\
\hline 256 & $78 \mathrm{kHz}$ & Sweep time $(s)$ & 66 & 52 & 37 \\
\hline 512 & $39 \mathrm{kHz}$ & 1 work-cycle $(\mathrm{ms})$ & 3,84 & 5,25 & 7,81 \\
\hline 512 & $39 \mathrm{kHz}$ & Sweep time $(s)$ & 96 & 68 & 53 \\
\hline 1024 & $19.5 \mathrm{kHz}$ & 1 work-cycle $(\mathrm{ms})$ & 6,71 & 7,94 & 10,5 \\
\hline 1024 & $19.5 \mathrm{kHz}$ & Sweep time $(s)$ & 162 & 98 & 68 \\
\hline 2048 & $10 \mathrm{kHz}$ & 1 work-cycle $(\mathrm{ms})$ & 13,79 & 14,28 & 17,09 \\
\hline 2048 & $10 \mathrm{kHz}$ & Sweep time $(s)$ & 325 & 172 & 106 \\
\hline 4096 & $4.9 \mathrm{kHz}$ & 1 work-cycle $(\mathrm{ms})$ & $\mathrm{X}$ & 25,88 & 28,56 \\
\hline 4096 & $4.9 \mathrm{kHz}$ & Sweep time $(s)$ & $X$ & 305 & 172 \\
\hline \hline
\end{tabular}

Processed data are finally stored in an ASCII database and handled by off-line software to produce an RFI map.

Two monitoring modes are possible:

- Scanning of the whole band $0.1-2.35 \mathrm{GHz}$ at low system time efficiency;

- Scanning of different radio bands of interest for radio astronomy at a very high system time efficiency.

In the first case a complete overall RFI scenario is obtained. In the second one an RFI scenario within the programmed bands is given. The System is able to detect a very wide variety of RFI with levels spanning from $-51 \mathrm{dBm}$ up to $14 \mathrm{dBm}$ (measured at the IF output). Weak RFI needs long integration time, while military spread spectrum signals need wide bands and very short integration time.

\footnotetext{
${ }^{1}$ The higher the burst-size, the faster the system throughput (execution time for a single workcycle), because there is a fixed time for board management and control that loses relevance as the board memory is exploited.
} 


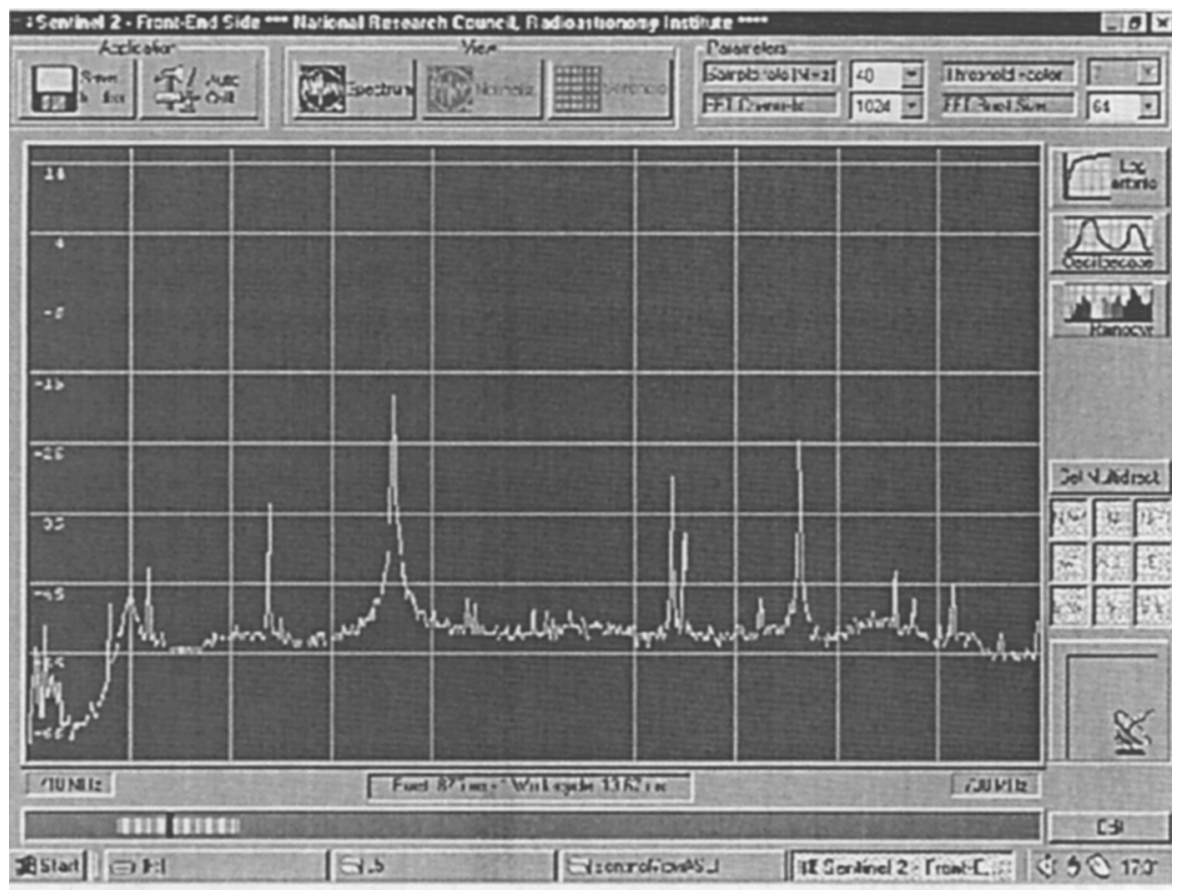

Figure 4. On-line Processing and Control Graphical User Interface.

Here are the main characteristics of the Sentinel 2 system:

\begin{tabular}{|l|c|}
\hline Scanning window size (tuning step) & $20 \mathrm{MHz}$ \\
Dynamic Range: & {$[-52,+14] \mathrm{dBm}$} \\
Total frequency range & {$[100,2350] \mathrm{MHz}$} \\
\hline
\end{tabular}

- User-friendly on-line control and flexible off-line analysis software

- Modularity

- Less expensive than the commercial monitoring systems

\section{The Software}

Software for the Sentinel 2 control and off-line processing was designed and developed ad hoc under the Microsoft Visual Studio 6.0 environment. Care was taken to develop a flexible, fast and user-friendly package. We have two applications:

- On-line Processing \& Control;

- Off-line Spectrometer. 


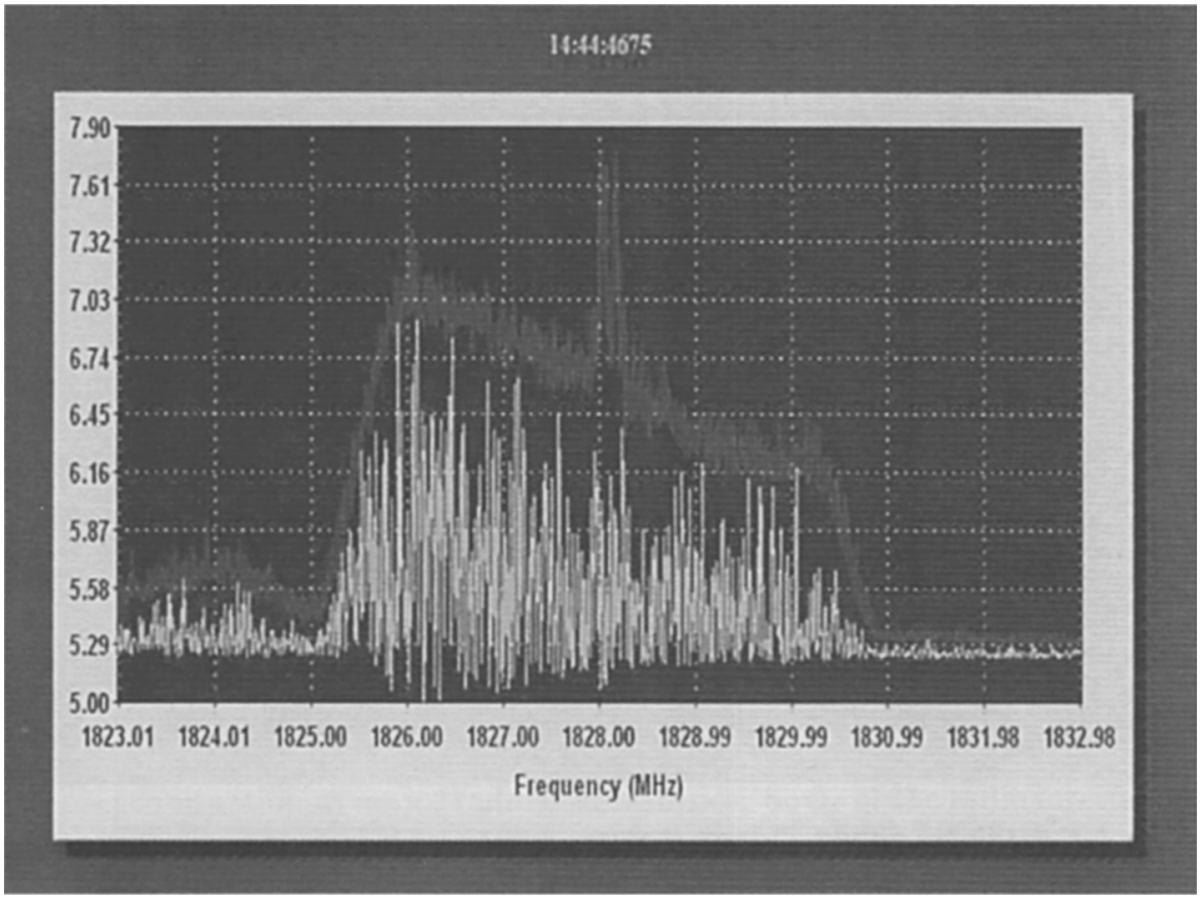

Figure 5. Spread-Spectrum Military Interference: The red (upper) plot represents the maximum channel value over the time period while the yellow plot represents a single spectrum (at 14:44:4675).

The first one is an on-line application in charge of the frontend/backend control, computing power spectra, averaging and data saving. It works under the Windows NT 4.0 operating system and was developed in the Microsoft Visual $\mathrm{C}^{++}$ 6.0 environment. The graphical interface (see Figure 4) allows the user both to easily programme the working parameters and to control the system activity.

The second application works off-line. It reads spectra from the database, providing graphical tools for better display. It operates under Windows $95 / 98$ or NT 4.0 and it was developed under Microsoft Visual Basic 6.0 using the Olectra Chart 6.0 ocx library.

A miscellany of different kinds of RFI processed by S2 is illustrated in Figures 5, 6 and 7. 


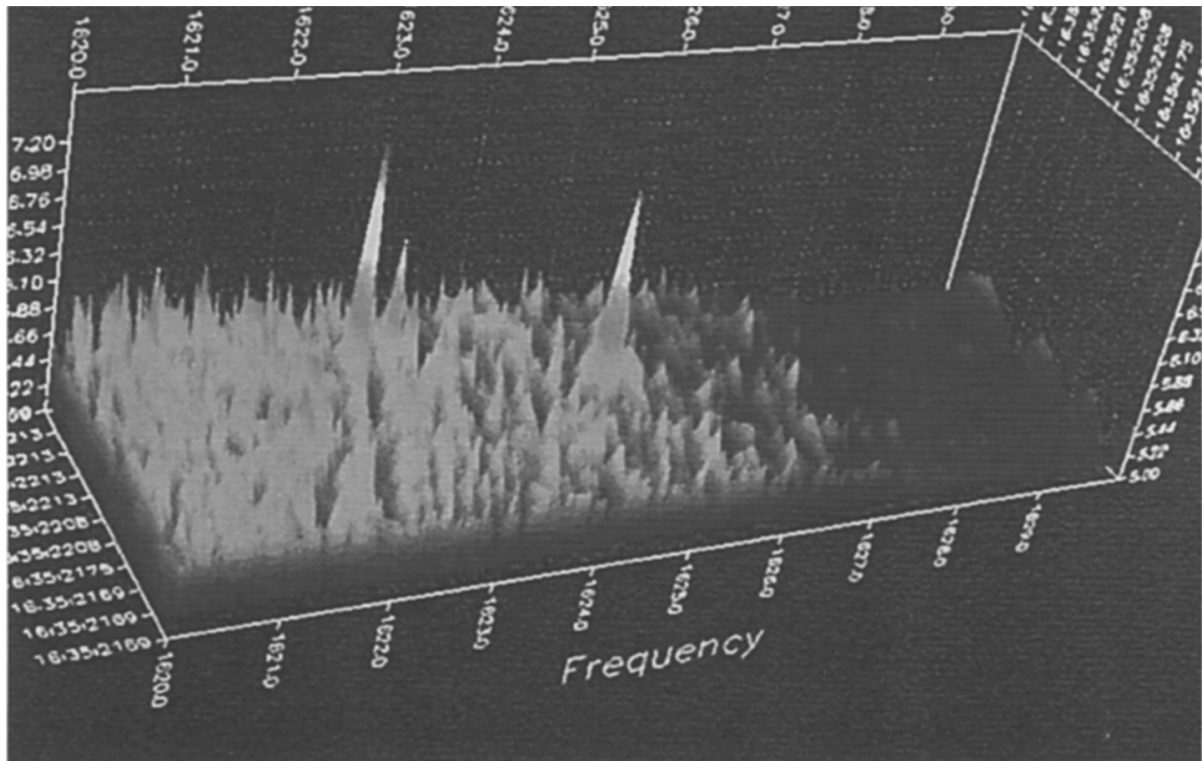

Figure 6. Iridium Interference at $\sim 1620 \mathrm{MHz}$ over $320 \mathrm{msec}$ of acquisition (3-D view).

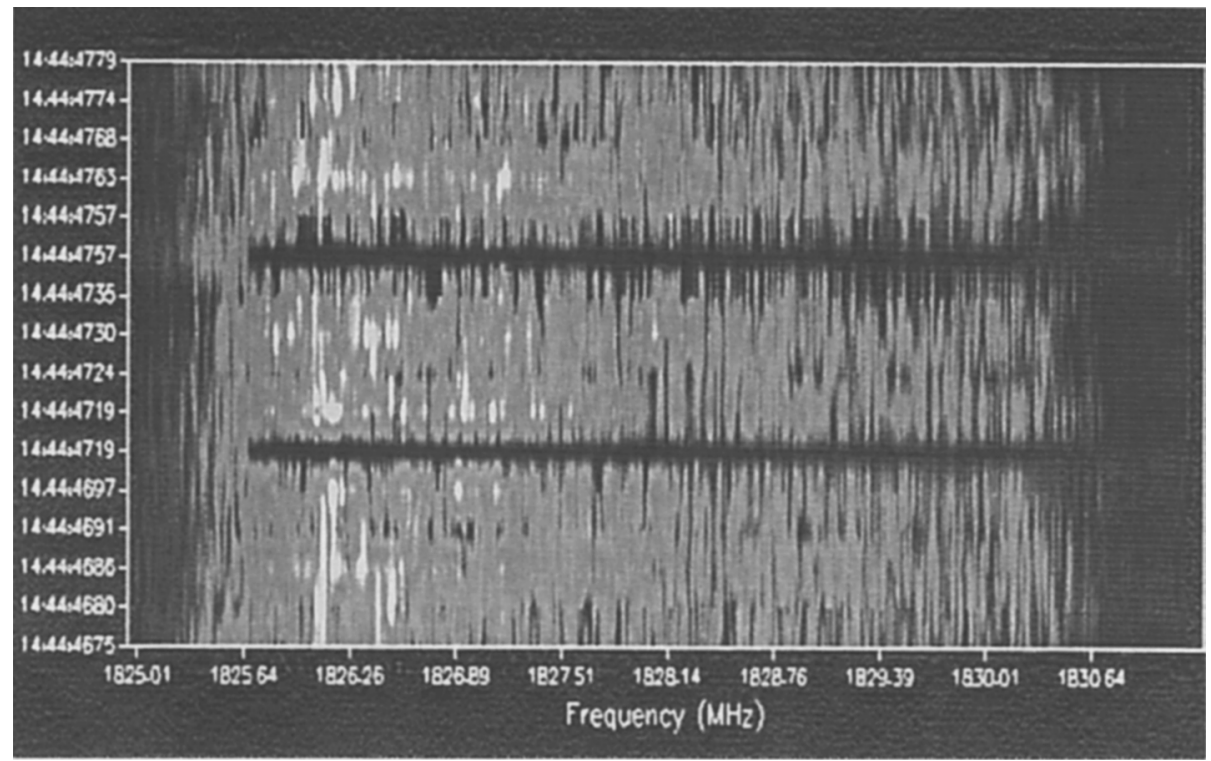

Figure 7. The same interference displayed in a Time-Frequency chart. The colour represents the intensity of the signal. 